\title{
Development of a Korean-Type Qualitative Case History Tinnitus Questionnaire
}

\author{
Se-Joon $\mathrm{Oh}^{1}$, Eui-Kyung Goh ${ }^{1}$, Soo-Keun Kong ${ }^{1}$, Shi Nae Park ${ }^{2}$, \\ June Choi ${ }^{3}$, Hyun Min Lee ${ }^{4}$, Seung Chul Ha ${ }^{5}$, and Il-Woo Lee \\ ${ }^{I}$ Department of Otorhinolaryngology-Head and Neck Surgery, Pusan National University School of Medicine, \\ Pusan National University Hospital, Busan; and ${ }^{2}$ Department of Otolaryngology-Head and Neck Surgery, \\ The Catholic University of Korea College of Medicine, Seoul; and ${ }^{3}$ Department of Otorhinolaryngology-Head and Neck Surgery, \\ Korea University College of Medicine, Seoul; and ${ }^{4}$ Department of Otorhinolaryngology-Head and Neck Surgery, \\ Pusan National University School of Medicine, Pusan National University Yangsan Hospital, Yangsan; and \\ ${ }^{5}$ Department of Otolaryngology, Asan Medical Center, University of Ulsan College of Medicine, Seoul, Korea
}

\author{
한국형 정성적 병력 이명 설문지의 개발 \\ 오세준 ${ }^{1} \cdot$ 고의경 ${ }^{1} \cdot$ 공수근 $^{1} \cdot$ 박시내 $^{2} \cdot$ 최 준 $^{3} \cdot$ 이현민 $^{4} \cdot$ 하승철 $^{5} \cdot$ 이일우 $^{4}$ \\ 부산대학교 의과대학 부산대학교병원 이비인후과학교실, ${ }^{1}$ 가톨릭대학교 의과대학 이비인후과학교실, ${ }^{2}$ \\ 고려대학교 의과대학 이비인후과학교실, ${ }^{3}$ 부산대학교 의학전문대학원 양산부산대학교병원 이비인후과학교실, ${ }^{4}$ \\ 울산대학교 의과대학 서울아산병원 이비인후과학교실
}

\author{
Received July 25,2017 \\ Revised August 29, 2017 \\ Accepted September 13, 2017 \\ Address for correspondence \\ Il-Woo Lee, MD, PhD \\ Department of Otorhinolaryngology- \\ Head and Neck Surgery, \\ Pusan National University \\ School of Medicine, \\ Pusan National University \\ Yangsan Hospital, \\ 20 Geumo-ro, Mulgeum-eup, \\ Yangsan 50612, Korea \\ Tel +82-55-360-2132 \\ Fax $+82-55-360-2162$ \\ E-mail entgate@gmail.com
}

Background and Objectives Qualitative and quantitative data of tinnitus are both important to obtain necessary information for assessing tinnitus evaluation. But contrary to quantitative questionnaire, qualitative data is not standardized in Korea. This study aimed to standardize the qualitative data of tinnitus by developing a Korean-type integrated qualitative tinnitus questionnaires.

Subjects and Method A cross-sectional survey of Korean otolaryngologists was performed. The questionnaires were administered to otologists who were registered as participants in the tinnitus study group of otology research interest group (ORIG).

Results Most of the otologists (100\% of responders) have used the quantitative tinnitus questionnaire measurement $(90.5 \%)$, but only $76 \%$ have used qualitative tinnitus questionnaire. From the responses of otologists who regularly use qualitative questionnaire, 25 items were adopted from the 35 item list. Questionnaire items were selected according to the frequency of listed items in the individual lists.

Conclusion We made a qualitative questionnaire consisting of 25 items that were essential and widely accepted. We expect this work will integrate and standardize qualitative tinnitus questionnaires in Korea. Korean J Otorhinolaryngol-Head Neck Surg 2018;61(4):182-92

Key Words Korea - Qualitative research - Questionnaire · Tinnitus.

\author{
서 론 \\ 이명(tinnitus)은 일반적으로 외부 자극 없는 상태에서 소리 \\ This is an Open Access article distributed under the terms of the Creative Commons \\ Attribution Non-Commercial License (http://creativecommons.org/licenses/by-nc/4.0) \\ which permits unrestricted non-commercial use, distribution, and reproduction in any \\ medium, provided the original work is properly cited.
}

가 들린다고 느끼는 이상 음감을 의미한다. 많은 사람들이 간헐적인 이명을 경험한다고 알려져 있는데, 성인의 약 $10 \%$ 에서 5 분 이상 지속되는 이명을 보이고 이 중 $1 \%$ 는 이명으로 인하여 심한 불편감, 수면장애 및 짜증 등의 증상을 호소하 기도 한다. ${ }^{1)}$ 하지만 현재까지 알려져 있는 다양한 치료방법의 효과에 대해서는 합치된 의견이 아직 없는 실정이다. ${ }^{2)}$ 그럼에 
도 불구하고 이명으로 인한 불편감이나 치료 효과에 대한 검 증을 위한 도구는 반드시 필요하다. 이명은 다양한 형태와 특 징을 가지고 있으나, 타인에게 명확하게 인지되지 않으며 이명 의 진단을 위한 객관적인 진단법조차 아직 개발되어 있지 않 다. 따라서 대부분 환자가 호소하는 증상을 듣고 환자가 이명 을 호소하는지, 어떤 형태인지를 알아낼 수 있다. 모든 의료 영 역에서 병력(case history)은 중요하지만, 주로 주관적인 증상 으로 평가하는 이명에서는 특히 의미가 있다. 더구나 이명의 주관적인 특징으로 인해 이명 환자의 치료에서뿐만 아니라 연구에서의 적용은 쉽지 않아서 모든 이명 환자에서는 청각 학적, 이과적인 평가와 더불어 자세한 병력 청취가 치료방법 에 대한 정보를 획득하는 데 필요하다.

주관적 이명의 경우 이명 강도와 주파수를 객관적으로 측정 하기 어렵기 때문에 치료 전후 이명의 평가를 위해 각종 설문 지가 사용되고 있다. 사용되고 있는 이명 설문지는 크게 정성 적(qualitative)인 설문지와 정량적(quantitative)인 설문지로 나눌 수 있다. 정량적 설문지는 Tinnitus Handicap Inventory (THI)가 가장 널리 사용되고 있으며 한국어판으로도 번역되 어 있고 이미 신뢰도 및 타당도가 검증되어 있다.") $\mathrm{THI}$ 는 정 량적 검사로써 이명의 등급을 매기거나 임상적인 치료, 연구 (research) 등에는 적용하기에 유용하지만, 환자 각자의 이명 의 성상을 자세히 파악하는 데는 한계가 있어 이런 단점을 보 완하기 위해 정성적인 접근을 함께하는 것이 필요하다. 현재 여러 개의 정성 병력 설문지가 개발되어 있으나 아직 많은 임상 의들은 본인이 중요하고 이명과 관련이 있다고 여기는 설문 지를 각자 개발하여 사용되고 있는 실정이다. ${ }^{4-6)}$

본 연구의 목적은 현재 우리나라의 대학병원 및 이비인후과 전문병원에서 이명 평가에 사용되는 도구에 대한 설문조사 를 통해 정량적, 정성적 설문지를 포함하여 이명 환자의 평가 실태를 확인하고, 그 내용을 바탕으로 표준화된 정성적 병력 이명 설문지를 개발하여 대학병원뿐만 아니라 우리나라의 어느 병원에서나 사용할 수 있도록 하였다. 그렇게 함으로써 이명 환자의 평가방법에 대한 합의를 이루고 임상 정보를 공 유하여 더 많은 치료 정보를 얻는 데 도움이 되고자 하였다.

\section{대상 및 방법}

본 연구에 앞서 이비인후과 및 산업의학에 근무하는 9인의 전문가로 구성된 대한이과학회 산하 Otology Research Interest Group(ORIG)의 이명연구회 내 이명 설문지 소위원회를 설문지에 포함할 내용과 정성적 설문지에 포함할 수 있는 많 은 항목에서 중요성이 높은 항목을 선정하여 한국형 정성 이 명 설문지를 개발하기 위한 위원회 모임을 가졌다. 본 횡단적
단면연구는 크게 6 개의 문항으로 구성되었고, 이명 설문지 평가 유무, 정량적 및 정성적 이명 설문지 사용 유무 및 실태, 정성적 평가 설문지에 포함되는 내용에 대해 질문을 하였으 며, 그 외 추가로 visual analogue scale(VAS) score 중 사용 하는 항목과 청각과민, 우울증, 스트레스, 불안증 등의 증상 에 대한 대한 내용도 포함되었다(Appendix I). 마지막으로 상 기의 이명 설문지 외에 다른 내용의 설문을 하고 있는지, 이 를 치료 시 반영하는지에 대한 질문이 추가되었다. 정성적 평 가 설문지에 포함되는 내용에 대한 질문에는 2006년 Regensburg에서 열린 이명 컨센서스 워크숍에서 발표된 이명 병력 설 문지를 위한 “items list"를 참고로 하였는데 여기에는 필수항 목으로 여겨지는 'A' 항목이 14 개, 포함시키는 것이 바람직하 다고 평가되는 'B' 항목이 21 개의 요소로 모두 35 개의 항목 으로 구성되어 있다. ${ }^{7)}$ 35개의 설문 항목에서 각 병원에서 선 택한 항목을 분석하여 중요도와 빈도 순을 고려하여 한국형 정성 이명 설문지를 개발하고자 하였다. 개발된 정성적 설문 지의 각 항목들은 일차적으로 2 명의 이과 전문의에 의해 번 역이 시행되었다. 번역에서 일반적인 중등 교육 과정을 이수 한 사람이 스스로 내용을 이해하기에 어려움이 없을 정도의 한국어 단어로 번역하는 것이 목표였으며, 어휘 및 관용구에 대한 검토는 표준국어대사전을 활용하였다. 설문지의 번역은 이해도를 높이기 위해 대한민국의 실생활에 흔히 쓰이는 자 연스러운 문장이 되도록 의역하는 방식을 채택하여 한국형 정성 이명 설문지를 개발하였다(Appendix II).

\section{결 과}

\section{연구 참가자}

설문조사는 대한이과학회 이명 분과 모임의 참여 회원으 로 등록된 전국 22 개 대학병원 및 이비인후과 전문병원의 이 비인후과 전문의들을 대상으로 시행되었다. 각 병원당 한 명의 전문의에게 전자우편을 통해 설문지를 보내었고, 설문조사 의 응답률은 $100 \%$ 였다.

\section{이명 설문지}

정량적인 이명 설문지 평가를 하고 있는 병원은 22개 병원 중 21개 병원이었다(90.5\%). 그중 19개 병원(90\%)에서 THI가 사용되고 있었고, Tinnitus Handicap Questionnaire(THQ), Subjective Tinnitus Severity Scale(STSS)을 각각 1개의 병 원에서 사용하고 있었다. 하지만 정성적 평가를 함께 시행하 는 병원은 16 개로 $76 \%$ 였다. 이 중 정성적 평가용 설문지를 자체적으로 제작하여 사용하는 경우가 12 개(57\%) 병원이었 고, 다른 병원의 것을 사용하는 경우가 3 개(14\%)였으며 6개 
병원은 무응답(28\%)하였다. "Item list" 35개 항목에 대해 각 병원에서 사용 중인 항목을 선택하도록 하였을 때 답변을 한 병원은 22개 병원 중 14 개 병원이었고(63.6\%), 그 선택률은 다음과 같다(Table 1). 모든 병원에서 선택한 항목에 대해 응 답률을 분석하고, 최종적으로 $50 \%$ 이상의 응답을 보인 항목 과 필수항목으로 여겨지는 'A' 항목을 합하여 35개의 문진 항목 중 최종 25 개가 선정되어 이를 이명 병력 설문지로 개발
하였다(Table 2). 10-scale VAS를 사용하여 설문하는 경우는 7개 병원(33\%)에서 시행하고 있다고 답하였다. 이명 설문지 평가의 빈도에 대한 질문에서 중복 답변을 포함하여 21 개 병 원에서 답변을 하였고, 첫 내원 시에만 평가하는 경우가 5개 병원(24\%), 병원을 내원할 때마다 평가하는 경우가 2개 병원 (10\%), 일정 간격을 두고 평가하는 경우가 9개 병원(43\%)이 었으며 이 중 6 개 병원은 정확한 기간에 대해서는 응답하지

Table 1. Percentage of acceptance of each hospital questionnaire

\begin{tabular}{|c|}
\hline Background (\%) \\
\hline 1. Age \\
\hline 2. Gender \\
\hline 3. Handedness \\
\hline 4. Family history of tinnitus (parent, children) \\
\hline Tinnitus history (\%) \\
\hline 5. Initial onset. Time? \\
\hline 6. Initial onset. Mode? Gradual or abrupt? \\
\hline $\begin{array}{l}\text { 7. Initial onset. Associated events? Hearing change } \\
\text { dental treatment, stress other. }\end{array}$ \\
\hline 8. Pattern. Steady? Pulsatile? Other? \\
\hline 9. Site. Right ear? Left ear? Both ears? (symmetrical? \\
\hline 10. Intermittent or constant? \\
\hline 11. Fluctuant or non-fluctuant? \\
\hline 12. Loudness. Scale 1-100. At worst \& at best? \\
\hline 13. Quality. Own words/give a list of choices. \\
\hline 14. Pure tone or noise? Uncertain/polyphonic? \\
\hline 15. Pitch. Very high? High? Medium? Low? \\
\hline 16. Percentage of awake time aware of tinnitus? \\
\hline 17. Percentage of awake time annoyed by tinnitus? \\
\hline 18. Previous tinnitus treatments (no, some, many)? \\
\hline
\end{tabular}

Modifying influences (\%)

19. Natural masking? Music, everyday sounds, other sounds?

20. Aggravated by loud noise? 71

21. Altered by head and neck movement or touching of head or upper limbs (specification of the respective 43 movements)?

22. Daytime nap. Worse? Better? No effect? 21

23. Effect of nocturnal sleep on daytime tinnitus? 29

24. Effect of stress?

25. Effect of medications? Which?

Related conditions (\%)

26. Hearing impairment?

27. Hearing aids (no, left ear, right ear, both ears; effect on tinnitus)?

28. Noise annoyance or intolerance?

29. Noise induced pain? 29

30. Headaches?

31. Vertigo/dizziness?

32. Temporomandibular disorder?

33. Neck pain? 14

34. Other pain syndromes?

35. Under treatment for psychiatric problems? 29

Category "A" (=essential) in bold type. The items 1, 2, 4, 5, 6, 8, 9, 12, 16, 19, 21, 26, 27, 28 are considered as essential (category A) all other items are regarded as highly desireable (category $B$ ) 
Table 2. Final qualitative questionnaire

1. 나이

2. 성별

3. 직계가족 중에 이명을 호소하는 사람이 있습니까?

4. 이명이 처음 발생한 시간으로부터 얼마나 지났습니까?

5. 이명이 처음 발생할 때 양상이 어떠하였나요? 서서히 또는 갑자기?

6. 이명이 처음 발생할 때 관련된 사건이 있었나요? 예를 들면, 청력저하, 소음, 중이염, 두부 외상, 상해, 치과 치료, 스트레스 등

7. 이명의 양상이 지속적인가요? 박동성인가요? 아니면 또 다른 양상인가요?

8. 이명이 어디에서 들리나요? 우측, 좌측? 또는 양측? 아니면 머릿속에서 들립니까?

9. 이명이 항상 들립니까? 아니면 들리지 않을 때가 있습니까?

10. 이명이 일정한가요? 아니면 변동적입니까?

11. 이명의 크기(강도)를 점수로 매긴다면(1 100) 가장 좋을 때와 가장 나쁠 때의 점수는?

12. 이명이 어떤 소리인지 자신만의 단어로 표현해 보세요(벨 소리, 귀뚜라미 소리, 음악, 쉿쉿, 휘파람 소리, 변압기 소리, 맑은 소리, 윙윙, 딸칵, 높은 장력을 지닌 철사 소리, 맥박 뛰는 소리, 지글지글 소리, 쿵쿵).

13. 이명이 순음(단일 소리)인가요? 잡음인가요? 아니면 분명치 않거나 여러 가지 소리가 들리나요?

14. 이명의 높이는 어떻습니까?(아주 높다, 높다, 중간이다, 낮다)

15. 깨어있을 때 이명을 느끼는 시간의 비중은 얼마나 되나요?(\%)

16. 깨어있을 때 이명으로 괴로운 시간의 비중은 얼마나 되나요?(\%)

17. 본원 내원 전에 이명 치료를 받은 적이 있습니까?(없다, 조금, 많다)

18. 일상 환경 음에서 이명이 약해지는 소리가 있습니까?(음악, 일상음, 기타)

19. 이명이 큰 소음에 의해 악화됩니까?

20. 머리와 목을 움직이거나 머리나 상지를 잡으면 이명이 변화합니까?

21. 스트레스가 이명에 영향을 미칩니까?

22. 어떤 약물 치료가 이명에 영향을 미칩니까?

23. 난청이 있습니까?

24. 보청기를 사용 중입니까?(아니오, 우측, 좌측, 양측; 이명에 영향이 있습니까?)

25. 소음이 성가시거나 견디기 힘듭니까?

Category "A" (=essential) in bold type. The items 1, 2, 3, 4, 5, 7, 8, 11, 15, 18, 20, 23, 24, 25 are considered as essential (category A) all other items are regarded as highly desireable (category B)

않았고, 2개 병원에서 3개월마다, 1개 병원에서 2개월마다 평 가하였다. 그리고 5개 병원(24\%)은 불규칙적으로 평가를 하 였고, 2개 병원(10\%)은 치료방법이 바뀌거나 오랜만의 재방 문 시 설문지를 조사하였다. 반복적으로 이명 설문지 평가를 하는 경우에는 중복 답변을 했을 때, 정량적 설문지는 16 개 (80\%)로 가장 많았고, VAS score는 14 개(70\%), 정성적 설문 지 3개 $(15 \%)$ 순이었다. 이명 설문지에서 추가 질문은 청각과 민 7개(58\%) 병원으로 가장 많았고, 우울증 6개(50\%), 스트 레스 3 개 $(25 \%)$, 불안증 3 개 $(25 \%)$ 병원 순이었고, 그 밖에 1 개 병원에서 수면에 관한 질문을 하고 있었다. 마지막으로 상 기 이명 설문지 외에 설문을 하고 있는 경우 이를 치료에 반 영하는 병원은 14 개 병원(64\%)이었고, 8 개 병원(36\%)은 반영 하지 않았다.

\section{고 찰}

본 연구에서 우리나라에서는 현재 이명 설문지의 경우 $90 \%$ 이상의 병원에서 이명 환자의 평가에 있서 이명 설문지가 이용
되고 있으며, 정량적인 평가에서는 $90 \%$ 에서 $\mathrm{THI}$ 로 시행하 였으므로 대부분의 병원에서 동일한 정량적인 평가를 하고 있는 것으로 나타났다. 하지만 정성적 평가용 설문지는 사용 하고 있는 경우가 $76 \%$ 로써 정량적인 설문지에 비해 사용률이 떨어졌고, 포함되는 내용 또한 다양하였다. 이는 아직 한국에 표준화된 정성 설문지가 없는 것이 원인이라 생각된다. 정량적 으로 평가할 수 있는 항목이 다양하므로 설문지를 만들기가 쉽지 않으며 의사의 개인적인 중요성에 따라 선택된 항목은 다양할 수밖에 없었다. 따라서 본 연구에서는 정량적 설문지 인 THI와 같이 한국에서 많은 병원에서 이용할 수 있는 한 국형 정성적 이명 설문지를 만드는 것을 목표로 하였다. 이를 위해 저자들은 2006년 독일의 Regensburg에서 제시된 35개 항목으로 구성된 "item list"를 기본으로 이용하였는데, 이명 환자의 평가 및 결과 측정에서 컨센서스(consensus)를 얻기 위 해 만들어진 것으로써, 각 이명 전문가들이 여러 병력 설문지 에 공통적으로 포함되는 항목 및 중요하다고 간주하는 항목 들에 대한 합의가 이루어져 선택되었다. ${ }^{5)}$

각 항목은 임상적으로 중요한 내용이지만 이명의 정량적 평 
가를 위한 THI의 25개 항목도 함께 시행한다는 점을 고려한 다면 설문 시간이 너무 길어지는 단점이 있을 수 있다. 즉, 약 10 15분 정도 소요되는 THI에 상대적으로 더 많은 시간이 소요될 수 있는 정량적 평가가 추가된다면 30분가량이 소요 될 수 있어 주로 많은 노인 환자들에서는 협조가 떨어질 수 있 다. 따라서 필수항목인 14 개 질문에, 본 연구에 포함된 병원 에서 시행하는 설문 내용에 $50 \%$ 이상 포함되는 항목 11 개를 포함하여 25개 항목으로 완성되었다. 14개의 필수항목들에 포함된 항목들은 임상적 중요성이 다른 항목에 비해 크기 때 문에 설문지에 포함을 하였고, 그 외 추가되는 11 개 항목에 각 병원에서 $50 \%$ 이상 포함되는 항목을 선택한 것은 항목 및 검사 시간을 줄이기 위해서는 불가피할 방법으로 사료된 다. 하지만 본 연구에서 완성된 설문지는 환자 개인마다의 상 황이 고려되지 않은 핵심 목록이므로 임상의가 특별히 더 중 요하다고 생각하는 항목이 있다면 추가할 수도 있다.

다음으로 이명 설문지를 이용하는 21 개 병원 중 초진에만 사용하는 경우가 5 개(24\%), 나머지 병원에서는 정기적, 부정기 적 또는 치료방법 변경 시에 설문지를 이용하였다. 설문지 빈 도에 대한 문헌상의 보고는 없으므로 가장 적합한 설문지 사 용에 대한 방법은 확인할 수 없었지만 대다수의 병원에서 연 속적으로 설문지를 통해 환자의 이명을 평가하고 있다는 것 을 확인하였다. 반복적인 설문지 이용 시에는 주로 정량적 설 문지와 이명에 대한 VAS score가 대부분이었으며 정성적 설 문지를 이용하는 경우는 $15 \%$ 에 불과하였다. 이는 정성적인 이 명 설문지는 초진 시 환자 이명 상태에 대한 평가에 유용하 므로 특별한 양상의 변화가 없는 한 시간적인 제약으로 방문 시마다 확인하는 것은 제한적이라는 현실적인 이유로 생각 된다. 한편, 환자의 괴로움 정도 등을 측정하는 데는 정량적 인 평가가 유용하므로 치료에 따른 괴로움의 변화를 확인하 는 데는 정량적인 평가로 추적관찰하는 것이 좋은 것으로 보 인다. 정성적 설문지가 없으므로 각 병원에서 중요시되는 증 상에 대한 추가적인 질문을 하는 병원들이 절반이 넘었고 주 로 청각과민 및 우울, 스트레스, 불안 등에 대한 질문이 있었 다. 이런 증상들은 치료에서 추가적인 약물이나 치료법이 적용 될 수 있기 때문으로 생각되며 실제로 약 $2 / 3$ 의 병원에서 이 를 치료에 반영하고 있는 것으로 밝혀졌다.

이명을 접근하는 데 가장 중요한 것은 정확한 진단 및 평가 로써 일반적으로 그 방법 중 하나로 설문지가 사용된다. 이명 설문지는 검사 시간이 짧을수록 좋으며, 간단하면서 필수적 인 내용을 포함해야 한다. 설문의 질을 높이기 위해 치료자가 직접 환자와 함께 작성하는 것이 도움이 되고 반복 측정, 설 문 배열을 변경할 수 있다. 이런 이명 설문지를 개발하기 위해 서는 하나의 의료기관이 아닌 전국적인 합의(nationwide con- sensus)가 필요한데, 본 연구에는 전국 주요 병원기관이 참여 하였기 때문에 전국적인 합의가 이루어졌다고 생각할 수 있 다. 이명 설문지는 크게 정량적인 평가를 위한 변별 설문(discriminative questionnaire)과 정성적인 방법인 병력(case history)을 통한 평가적 설문(evaluative questionnaire)이 존재하 며 이명의 평가에서 정성적, 정량적 설문지가 모두 필요하다.

정량적인 이명 설문지는 이명의 불편하고 심한 정도를 접근 하는 데 사용할 수 있다. 일반적으로 이명으로 병원을 방문하 는 환자들은 정도의 차이는 있지만 이명에 대한 고통을 동반 하고 있다. 즉, 어느 정도 습관화를 이루고 있는 환자부터 일 상 생활에 심각한 고통을 갖고 있는 환자도 존재하지만 그 정 도를 반영할 수 있는 척도가 필요하다. 이명의 크기 혹은 높 낮이가 이명에 의한 고통을 예측할 수 없으며, 오히려 이명의 괴로움, 장애 및 제약을 직접적으로 정량화하는 것이 이명에 의한 고통을 측정하는 데 더 적합한 것으로 알려졌다. ${ }^{8)} \mathrm{THI}$ 는 가장 널리 사용되고 있는 정량적인 설문지인데 후속 연구 에서 신뢰도 및 타당도가 충분히 검증되어 있다."

일반적으로 이명에 대한 정성적인 정보는 두 가지 방법으로 얻을 수 있다. 첫째는 병력 설문지(case history questionnaires) 이고 둘째로는 구조화된 면담(structured interview)이다. 구 조화된 면담을 시행하는 목적은 이명의 원인, 진단적인 변수 들을 조사하여 환자의 이명의 성상을 철저히 이해하고자 하 는 것이다. 그리하여 이러한 과정을 통해 얻어진 정보는 이과 적, 청각학적 접근과 더불어 이명의 진단과 치료를 위한 기본 토대가 된다. 면담을 시행할 때에는 이명의 병력 및 기술적인 (descriptive) 특징, 특징적인 행동, 사회적, 대인간 및 감정에 대한 영향, 이명을 악화시키거나 줄이는 요인들, 이전의 이명 치료 유무, 연관된 동반 질환 등을 철저하게 조사해야 한다. 하지만 임상의들은 많은 환자 수를 진료하는 우리나라 현실 에서 환자들의 자가 보고서인 설문지를 더 선호하는데, 그 이 유는 병력 설문지는 표준화된 질문을 함으로써 믿을 만하고 완전한 정보를 주며, 더구나 면담에 비해 소요 시간이 적다 는 장점 또한 가지고 있기 때문이며 이것이 본 연구의 목적이 라고 할 수 있다. 비록 병력 설문지가 이명 환자에 대한 정보 를 얻는 데 유용한 도구이기는 하지만 철저한 임상적인 면담 을 완전히 대체할 수는 없다. 따라서 병력 설문지를 시행하더 라도 환자와 상담을 통해 한 번 더 추가적인 평가는 항상 필 요하다. 환자의 이명에 대한 구체적인 양상을 알기 위해 정성 적인 이명 설문지가 필요하며, 본 연구를 통해 만들어진 정성 적 병력 이명 설문지를 통해 대한민국에서 표준화된 정성적 설문지를 이용하는 것이 도움이 되리라 생각된다.

이명에는 여러 가지 형태가 있으며 각각의 치료 또한 다르 다. 환자가 호소하는 이명을 정확하게 진단하고 완전하게 이 
해하기 위해서는 환자에 대한 문진이 가장 중요한데, 정성적 및 정량적 이명 설문지로 진료 시간을 단축할 수 있다. 이미 표준화된 정량적 설문지인 $\mathrm{THI}$ 외에 본 연구에서 전국 22개 병원에서 설문을 통해 정성적 설문지를 개발하였고 이를 통 해 이명 환자 접근에 더 용이해질 수 있을 것이다.

\section{Acknowledgments}

We thank the members of Otologic Research Interest Group of Tinnitus and The Korean Otologic Society for their support and participation in this study.

\section{REFERENCES}

1) Vesterager V. Tinnitus--investigation and management. BMJ 1997; 314(7082):728-31

2) Meikle MB, Henry JA, Griest SE, Stewart BJ, Abrams HB, McArdle $\mathrm{R}$, et al. The tinnitus functional index: development of a new clinical measure for chronic, intrusive tinnitus. Ear Hear 2012;33(2):153-76.

3) Kim JH, Lee SY, Kim CH, Lim SL, Shin JH, Cung WH, et al.
Reliability and validity of a Korean adaptation of the tinnitus handicap inventory. Korean J Otorhinolaryngol-Head Neck Surg 2002;45(4): 328-34.

4) Stouffer JL, Tyler RS. Characterization of tinnitus by tinnitus patients. J Speech Hear Disord 1990;55(3):439-53.

5) Langguth B, Goodey R, Azevedo A, Bjorne A, Cacace A, Crocetti A, et al. Consensus for tinnitus patient assessment and treatment outcome measurement: Tinnitus Research Initiative meeting, Regensburg, July 2006. Prog Brain Res 2007;166:525-36.

6) Henry JA, Jastreboff MM, Jastreboff PJ, Schechter MA, Fausti SA. Assessment of patients for treatment with tinnitus retraining therapy. J Am Acad Audiol 2002;13(10):523-44.

7) Langguth B, Searchfield GD, Biesinger E, Greimel KV. Chap. 47 History and Questionnaires. In: Møller AR, Langguth B, De Ridder D, Kleinjung T, editors. Textbook of tinnitus. 1st ed. New York: Springer;2010. p.387-404.

8) Hiller W, Goebel G. Factors influencing tinnitus loudness and annoyance. Arch Otolaryngol Head Neck Surg 2006;132(12):1323-30.

9) Newman CW, Sandridge SA, Jacobson GP. Psychometric adequacy of the Tinnitus Handicap Inventory (THI) for evaluating treatment outcome. J Am Acad Audiol 1998;9(2):153-60. 


\section{Appendix I}

I. 이명 환자에게 이명 설문지 평가를 하십니까?

1) 시행하지 않는다.

2) 시행한다.

3) 기타 - (

II. 귀하의 병원에서 사용하는 이명 설문지는 어떤 내용을 포함하고 있습니까?(이명 설문지란 실제 외래에 비치되어 있거나, 전산상에 등록되어 이명 환자에게 routine으로 사용하고 있는 것을 말합니다.)

1. 이명의 정성적 평가(qualitative measurement)를 위한 설문지를 사용하고 있습니까?

1) 사용하고 있다 ( )

2) 사용하지 않는다 ( )

3) 기타 - (

2. 정성적 평가용 설문지는 어떤 것을 사용하고 있습니까? 15 개 병원 답변, 6 개 병원 무응답

1) 별도로 제작하여 사용한다.

2) 다른 병원의 것을 함께 사용한다.

3. 사용 중인 정성적 평가 설문지에 포함되는 내용을 모두 표시하여 주세요.

1) Age

2) Gender

3) Handedness

4) Family history of tinnitus (parent, children)

5) Initial onset. Time?

6) Initial onset. Mode? Gradual or abrupt?

7) Initial onset. Associated events? Hearing change, acoustic trauma, otitis, media, head trauma, whiplash, dental treatment, stress other

8) Pattern, steady? Pulsatile? Other?

9) Site. Right ear? Left ear? Both ear? (symmetrical? inside head?)

10) Intermittent or constant?

11) Fluctuant or non-fluctuant?

12) Loudness. Scale 1-100. At worst and at best?

13) Quality. Own words/give a list of choices.

14) Pure tone or Noise? Uncertain/polyphonic?

15) Pitch. Very high? High? Medium? Low?

16) Percentage of awake time aware of tinnitus?

17) Percentage of awake time annoyed by tinnitus?

18) Previous tinnitus treatments (no, some, many)?

19) Natural masking? Music, everyday sounds, other sounds?

20) Aggravated by loud noise?

21) Altered by head and neck movement or touching of head or upper limbs? 
22) Daytime nap. Worse? Better? No effect?

23) Effect of nocturnal sleep on daytime tinnitus?

24) Effect of stress

25) Effect of medications? Which?

26) Hearing impairment?

27) Hearing aids(no, left ear, right ear, both ears; effect on tinnitus)?

28) Noise annoyance or intolerance?

29) Noise induced pain?

30) Headaches?

31) Vertigo/dizziness?

32) TMJ disorder?

33) Neck pain?

34) Other pain syndromes?

35) Under treatment for psychiatric problems?

4. 10-scale VAS를 사용하여 설문하는 항목을 기술하여 주십시오.

예시) Tinnitus Questionnaire

5. 정량적인 이명 평가를 위하여 사용하는 설문지는 어떤 것입니까? (모두 골라 주십시오) 20개 병원 답변(괄호 안은 문항 수)

1) $\mathrm{TCQ}=$ Tinnitus Cognitions Questionnaire (26)

2) $\mathrm{TCSQ}=$ Tinnitus Coping Style Questionniare (33)

3) THI=Tinnitus Handicap Inventory (25)

4) $\mathrm{THQ}=$ Tinnitus Handicap Questionnaire (27)

5) $\mathrm{TH} / \mathrm{SS}=$ Tinnitus Handicap/Support Scale (36)

6) $\mathrm{TQ}=$ Tinnitus Questionnaire (52)

7) $\mathrm{TRQ}=$ Tinnitus Reaction Questionnaire (29)

8) TSS=Tinnitus Severity Scale (15)

9) STSS=Subjective Tinnitus Severity Scale (15)

10) TSS=Tinnitus Severity Scale (12)

III. 이명 설문지 평가의 빈도는 어떠합니까?(중복 답변 포함)

1) 첫 내원 시에만 평가한다.

2) 병원 내원할 때마다 평가한다.

3) 일정 간격을 두고( 개월마다) 평가한다.

4) 불규칙하게 평가한다.

5) 기타 - (

IV. 반복적인 이명 설문지 평가를 하신다면 어떤 항목을 하십니까?(전부 표시 부탁드립니다.)

1) 정성적 설문지

2) VAS score

3) 정량적 설문지

4) 기타 - ( ) 
Korean J Otorhinolaryngol-Head Neck Surg I 2018;61(4):182-92

V. 이명 설문지 외에 시행하고 계신 설문이 있으신지요?(전부 표시 부탁드립니다.)(중복 답변 포함)
1) 청각과민에 대한 설문
2) 우울증에 대한 설문
3) 스트레스에 대한 설문
4) 불안증에 대한 설문
5) 기타 - (

VI. 상기의 이명 설문지 외에 설문을 하고 계신다면 이를 치료 시 반영하시는지요?

1) 반영한다.

2) 반영하지 않는다.

3) 기타 - ( 


\section{Appendix II}

Items are ordered according to their level of significance:

Category "A" (=essential) in bold type.

\section{Background}

1. 나이

2. 성별

3. 어느 쪽 손을 주로 사용하십니까?

4. 직계가족 중에 이명을 호소하는 사람이 있습니까?

\section{Tinnitus history}

5. 이명이 처음 발생한 시간으로부터 얼마나 지났습니까?

6. 이명이 처음 발생할 때 양상이 어떠하였나요? 서서히 또는 갑자기?

7. 이명이 처음 발생할 때 관련된 사건이 있었나요? 예를 들면, 청력저하, 소음, 중이염, 두부 외상, 상해, 치과 치료, 스트 레스 등

8. 이명의 양상이 지속적인가요? 박동성인가요? 아니면 또 다른 양상인가요?

9. 이명이 어디에서 들리나요? 우측, 좌측? 또는 양측? 아니면 머릿속에서 들립니까?

10. 이명이 항상 들립니까? 아니면 들리지 않을 때가 있습니까?

11. 이명이 일정한가요? 아니면 변동적입니까?

12. 이명의 크기(강도)를 점수로 매긴다면(1 100) 가장 좋을 때와 가장 나쁠 때의 점수는?

13. 이명이 어떤 소리인지 자신만의 단어로 표현해 보세요(벨 소리, 귀뚜라미 소리, 음악, 쉿쉿, 휘파람 소리, 변압기 소리, 맑은 소리, 윙윙, 딸칵, 높은 장력을 지닌 철사 소리, 맥박 뛰는 소리, 지글지글 소리, 쿵쿵).

14. 이명이 순음(단일 소리)인가요? 잡음인가요? 아니면 분명치 않거나 여러 가지 소리가 들리나요?

15. 이명의 높이는 어떻습니까?(아주 높다, 높다, 중간이다, 낮다)

16. 깨어있을 때 이명을 느끼는 시간의 비중은 얼마나 되나요?(\%)

17. 깨어있을 때 이명으로 괴로운 시간의 비중은 얼마나 되나요?(\%)

18. 본원 내원 전에 이명 치료를 받은 적이 있습니까?(없다, 조금, 많다)

\section{Modifying influences}

19. 일상 환경 음에서 이명이 약해지는 소리가 있습니까?(음악, 일상 음, 기타)

20. 이명이 큰 소음에 의해 악화됩니까?

21. 머리와 목을 움직이거나 머리나 상지를 잡으면 이명이 변화합니까?

22. 낮잠을 자면 이명이 어떻습니까?(악화, 호전, 변화 없다)

23. 밤의 수면이 낮 동안의 이명에 영향을 미칩니까?

24. 스트레스가 이명에 영향을 미칩니까?

25. 어떤 약물 치료가 이명에 영향을 미칩니까? 


\section{Related conditions}

26. 난청이 있습니까?

27. 보청기를 사용 중입니까?(아니오, 우측, 좌측, 양측; 이명에 영향이 있습니까?)

28. 소음이 성가시거나 견디기 힘듭니까?

29. 소음을 들으면 통증이 있습니까?

30. 두통이 있습니까?

31. 어지럼증이 있습니까?

32. 턱관절 질환이 있습니까?

33. 경부 통증이 있습니까?

34. 기타 통증 질환이 있습니까?

35. 정신과적인 치료를 받고 있습니까? 